\title{
GREEN'S FUNCTIONS COUPLED TO SELF-DUAL MAXWELL FIELDS
}

\author{
MATTHEW L. GINSBERG
}

\begin{abstract}
A twistor construction is given which gives an explicit form for the spin $n / 2$ propagators for massless fields coupled to a self-dual Maxwell field.
\end{abstract}

0. Introduction. Twistors were introduced by Penrose in $1967[12,15]$ in an attempt to provide a new description of complexified Minkowski space which naturally emphasized the conformal invariance of various physical systems.

Twistor theory has had considerable success dealing with systems of this sort, and we will be concerned with two of these specifically:

(1) The discovery by Ward $[16,17]$ that various self-dual gauge fields can be described in terms of vector bundles over projective twistor space $\mathbf{P}$ and

(2) The realization, originally due to Penrose $[14,5]$ that solutions of the massless field equations, minimally coupled to the self-dual fields of (1), can be described as elements of certain cohomology groups on $\mathbf{P}$.

There have been many explicit uses of these constructions; a general description of self-dual Yang-Mills fields appears in [17] and has subsequently been used by Atiyah, Hitchin, Drinfeld and Manin to solve these equations on $S^{4}$ [2]. More recently still, Atiyah [1] has managed to use the cohomology correspondence described in the preceding paragraph to construct spin-0 Green's functions for instanton-type Yang-Mills fields.

Our aim in this paper is to generalize these results for self-dual Maxwell fields by giving an explicit construction of Green's functions of arbitrary spin coupled to the field. Our construction will be explicit on twistor space only; spacetime fields can easily be obtained from their twistor counterparts by contour integration $[11,16]$.

The plan of the paper is as follows: $\S 1$ contains a brief review of the ideas of Ward $[16,17]$ and Penrose $[14,5]$ which underlie the subsequent analysis. $§ 2$ extends some ideas of Eastwood [3] which will be needed later but which have unfortunately not appeared in the general literature thus far.

Green's functions are dealt with in $\$ \S 3$ and 4. Theorem 3.3 describes the free-space Green's functions in terms of simplicial cohomology on the product of twistor space and its dual. $\$ 4$ consists of an extension of Theorem 3.3 to Green's functions minimally coupled to self-dual electromagnetic fields. Concluding remarks are contained in $\$ 5$.

Received by the editors March 12, 1982.

1980 Mathematics Subject Classification. Primary 81C30: Secondary 83A05.

'Platnauer Junior Research Fellow, Brasenose College. Oxford. 
This paper is an extension of earlier work in [7] and [4], and the results it contains have been sketched by the author in an Oxford preprint [8]. The author would like to thank Mike Eastwood and Roger Penrose for many illuminating discussions.

Our notation will follow [5]. Related material and an introduction to twistor theory can be found in [11].

1. The Ward correspondence and the Penrose transform. Defining $\mathbf{M}$ (complexified compactified Minkowski space) to be the Grassmanian of 2-planes in twistor space $\mathbf{T}$, and $\mathbf{F}$ to be the flag manifold of lines within 2-planes in $\mathbf{T}$, we have the usual twistor diagram [5]

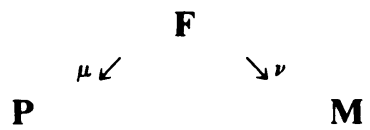

We will denote complexified Minkowski space by $\mathbf{M}^{\prime} \subset \mathbf{M}$.

If $\mathbf{T}^{*}$ is the dual of $\mathbf{T}$ and $\mathbf{P}^{*}$ is its projective version, we get a similar diagram

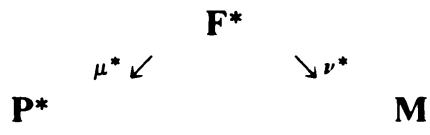

For a fixed region $U \subset \mathbf{M}$, we will define

$$
U^{\prime \prime}=\mu \nu^{-1}(U) \text { and } \quad " U=\mu^{*} \nu^{*-1}(U) .
$$

If, for example, $U$ is the forward tube

$$
\begin{aligned}
& \mathbf{M}^{+}=\left\{x^{a}-i y^{a} \in \mathbf{M}^{l} \text { such that } x^{a} \text { and } y^{a}\right. \text { are real } \\
& \text { and } \left.y^{a} \text { is timelike and future pointing }\right\},
\end{aligned}
$$

we have $\mathbf{M}^{+\prime}=\mathbf{P}^{+}$and $" \mathbf{M}^{+}=\mathbf{P}^{*-}$ (see [11], for example). Similarly for the backward tube

$$
\begin{aligned}
& \mathbf{M}^{-}=\left\{x^{a}-i y^{a} \in \mathbf{M}^{\prime} \text { such that } x^{a} \text { and } y^{a}\right. \text { are real } \\
& \text { and } \left.y^{a} \text { is timelike and past pointing }\right\},
\end{aligned}
$$

we have $\mathbf{M}^{-\prime \prime}=\mathbf{P}^{-}$and $" \mathbf{M}^{-}=\mathbf{P}^{*+}$.

Now suppose that $U, V \subset \mathbf{M}$. $U$ will be called suitable if:

(1) $U$ is Stein;

(2) $H^{1}(U ; \mathbf{Z})=H^{2}(U ; \mathbf{Z})=0$; and

(3) for any twistor $Z^{\alpha} \in U^{\prime \prime}, \nu \mu^{-1}\left(Z^{\alpha}\right) \cap U$ is connected and

$$
H^{1}\left(\nu \mu^{-1}\left(Z^{\alpha}\right) \cap U ; \mathbf{Z}\right)=0,
$$

and similarly for any dual twistor $W_{\alpha} \in{ }^{\prime \prime} U$.

$U$ and $V$ will be called null disjoint if $x \in U$ and $y \in V$ imply that $(x-y)^{2} \neq 0$, i.e., $x$ and $y$ are not null-separated.

Note that $\mathbf{M}^{+}$and $\mathbf{M}^{-}$are both suitable and are null disjoint.

The following result is essentially Ward's:

THEOREM 1.1 (WARD). Let $U \subset \mathbf{M}$ be suitable, and suppose that $E$ is a unitary vector bundle over $U$ with self-dual connection. Then $E$ lifts to a holomorphic vector bundle (which we will also denote by $E$ ) on $U^{\prime \prime}$. 
Self-dual electromagnetic fields, for example, correspond to line bundles over regions in $\mathbf{P}$.

We will denote by $\xi$ the hyperplane section bundle over $\mathbf{P}$, and will write $\theta(k)=\theta\left(\xi^{-k}\right)$ for the sheaf of germs of homogeneous functions on $\mathbf{P}$, homogeneous of degree $k$ in their argument. If $V$ is a vector bundle over a region in $\mathbf{P}$, we will abbreviate $\theta\left(V \otimes \xi^{-k}\right)$ to $V(k)$. Similarly, if $\xi^{*}$ is the hyperplane section bundle on $\mathbf{P}^{*}$, we write $\theta(k)=\theta\left(\xi^{*-k}\right), V(k)=\theta\left(V \otimes \xi^{*-k}\right)$ on $\mathbf{P}^{*}$, and $\theta^{*}(j, k)=$ $\theta\left(\xi^{-j} \otimes \xi^{*-k}\right), V(j, k)=\theta\left(V \otimes \xi^{-\prime} \otimes \xi^{*-k}\right)$ on $\mathbf{P} \times \mathbf{P}^{*}$.

Following [13], we will write $x^{A A^{\prime}}$ for a point in complexified Minkowski space $\mathbf{M}^{\prime}$, where the $A$ and $A^{\prime}$ are spinor indices. For a unitary vector bundle $E$ over a region $U \subset \mathbf{M}$, we will write $\nabla_{A}^{A^{\prime}}$ for the associated covariant derivative. In the electromagnetic case, we have [6]

$$
{ }_{E} \nabla_{A}^{A^{\prime}}=\frac{\partial}{\partial x_{A^{\prime}}^{A}}+2 \pi i \psi_{A}^{A^{\prime}} .
$$

where $\psi_{A}^{A^{\prime}}$ is a potential for the electromagnetic field. We define a massless field on $U$, of helicity $n / 2$ and minimally coupled to the bundle $E$, to be a spinor-valued symmetric holomorphic function $\phi$ on $U$ satisfying:

$$
\begin{aligned}
\nabla_{A}^{A_{A}^{\prime}} \phi_{A^{\prime} \cdots B^{\prime}}=0 & \text { for } n>0, \\
{ }_{E} \square \phi=0 & \text { for } n=0, \\
{ }_{E} \nabla_{A}^{A^{\prime} \phi^{A} \cdots B}=0 & \text { for } n<0,
\end{aligned}
$$

where $\phi$ has $|n|$ spinor indices and ${ }_{E} \square={ }_{E} \nabla_{A_{E}{ }^{\prime}} \nabla_{A^{\prime}}^{A}$. We will write ${ }_{E} Z_{n}$ for the group of all such massless fields. The basic result of [5] is the following:

Theorem 1.2 (Penrose). Let $U \subset \mathbf{M}$ be suitable, and suppose that $E$ is a unitary bundle over $U$ with self-dual connection. If $E$ is the lift of $E$ to $U^{\prime \prime}$ and $E^{*}$ its lift to " $U$, then there are natural isomorphisms

$$
H^{\prime}\left(U^{\prime \prime} ; E(-n-2)\right) \simeq{ }_{E} Z_{n} \simeq H^{1}\left({ }^{\prime \prime} U ; E^{*}(n-2)\right) .
$$

Theorems 1.1 and 1.2 are the natural starting point for this paper.

2. Nonprojective results. In this section we will discuss the relationship between the cohomology groups appearing in Theorem 1.2 and various cohomology groups on nonprojective twistor spaces. The results for the case where $E$ is the trivial line bundle were investigated by Eastwood in [3].

Let $E$ be a vector bundle over $U \subset \mathbf{P}$, and let

$$
\pi: \mathbf{T}-\{0\} \rightarrow \mathbf{P}
$$

be the usual projection. We will denote $\pi^{-1}(U)$ by $\tilde{U}$, and will similarly denote by $\tilde{E}$ the pullback of $E$ to $\tilde{U}$. Abusing notation, we will also denote the sheaves of germs of sections of $E$ or $\tilde{E}$ by $E$ or $\tilde{E}$, respectively.

Given a vector bundle $E$, it is possible to view the bundle $E(-1)$ as a complex manifold. $(\vartheta(-1) \simeq \mathbf{T}$, for example.) In the case where $E$ is a line bundle, this is Ward's "deformed twistor space", and there is natural identification of holomorphic functions on $E(1)$ with sections of the bundle $\tilde{E}$. 
The homogeneity operator on $\mathbf{P}$ is given by

$$
\Upsilon=Z^{\alpha} \frac{\partial}{\partial Z^{\alpha}},
$$

and since the transition functions for $E$ (and therefore $\tilde{E}$ ) are homogeneous of degree 0 ( $E$ being a holomorphic vector bundle over $\mathbf{P}$ ), it follows that $\Upsilon-n$ is a well-defined sheaf map from $\tilde{E}$ to itself. We therefore define sheaves $\tilde{E}(n)$ via the short exact sequence on $\mathbf{T}$ :

$$
0 \rightarrow \tilde{E}(n) \rightarrow \tilde{E}^{\Upsilon \stackrel{-}{\rightarrow}}{ }^{-} \tilde{E} \rightarrow 0 .
$$

Sections of $\tilde{E}(n)$ are simply "homogeneous" sections of the bundle $\tilde{E}$.

Theorem 2.1 (EASTwood [3]). For $U \subset \mathbf{P}$ and $E$ a vector bundle over $U$, there is an exact sequence

$$
\begin{aligned}
0 & \rightarrow H^{1}(U ; E(n)) \rightarrow H^{1}(\tilde{U} ; \tilde{E}(n)) \rightarrow H^{0}(U ; E(n)) \rightarrow H^{2}(U ; E(n)) \\
& \rightarrow H^{2}(\tilde{U} ; \tilde{E}(n)) \rightarrow H^{1}(U ; E(n)) \rightarrow \ldots
\end{aligned}
$$

Proof. The basic idea of the proof is to Laurent expand an arbitrary section of $\tilde{E}$ along the fibers of $\pi$ to evaluate the direct image sheaves $\pi_{*}^{j} \tilde{E}(n)$ and to then calculate the cohomology groups $H^{k}(\tilde{U} ; \tilde{E}(n))$ using the Leray spectral sequence $[10, \S I I .4 .17]$ and the generalized Gysin cohomology sequence [10, §I.4.6]. Details are in [3].

3. Uncoupled Green's functions. Suppose that $\Delta$ is some differential operator with no nontrivial global solutions. By a Green's function for $\Delta$, we will mean a kernel $G(x, y)$ for the inverse operator to $\Delta$.

Let $U \times V \subset \mathbf{M} \times \mathbf{M}$. It follows from Theorem 1.2 that a Green's function for the (uncoupled) differential operators appearing in (1.1), of helicity $n / 2$ in the first variable and $-n / 2$ in the second, will correspond to an element

$$
\phi_{n} \in H^{2}\left(U^{\prime \prime} \times^{\prime \prime} V ;(C(-n-2,-n-2))\right. \text {. }
$$

Suppose we define, for $n \geqslant 0$,

$$
(w \cdot z)_{n} \equiv \frac{(-1)^{n} n !}{4 \pi i\left(W_{\alpha} Z^{\alpha}\right)^{n+1}}
$$

and, for $U, V$ open subsets of a complex manifold $X$ and $\mathcal{S}, \mathcal{T}$ sheaves over $X$, an operation

$$
\cdot: H^{p}(U ; \varsigma) \otimes H^{q}(V ; \mathcal{T}) \rightarrow H^{p+q+1}(U \cup V ; \varsigma \otimes \Im)
$$

given by $\cdot=\partial^{*} U$, where $U$ is the usual cup product and $\partial^{*}$ is the Mayer-Vietoris coboundary $[7,4]$. It is then shown in [4] that the known spacetime properties of the Green's functions imply that, for $n \geqslant-1, \phi_{n}=(W \cdot Z)_{n+1} \cdot \phi$ for some fixed $\phi \in$ $H^{1}\left(\Omega_{U V} ; \theta\right)$ defined on a small neighborhood of $\Omega_{U V} \equiv\left\{W_{\alpha} Z^{\alpha}=0\right\} \cap\left(U^{\prime \prime} \times^{\prime \prime} V\right)$ in $\mathbf{P} \times \mathbf{P}^{*}$. It is shown in [7] and [9] that if $\zeta$ is any line bundle on $U^{\prime \prime} \times{ }^{\prime \prime} V$ with Chern class $(1,-1)$ such that the Chern class of the restriction of $\zeta$ to $\Omega_{U V}$ vanishes, we can take $\phi$ to be the pullback of this restriction along the map $e: \theta \rightarrow \theta^{*}$ given by $e(\zeta)=e^{2 \pi 1 \zeta}$. 
What we have done is considered the commutative diagram

$$
\begin{aligned}
& H^{1}\left(U^{\prime \prime} \times^{\prime \prime} V ; \vartheta\right) \rightarrow H^{1}\left(U^{\prime \prime} \times^{\prime \prime} V ; \vartheta^{*}\right) \stackrel{c}{\rightarrow} H^{2}\left(U^{\prime \prime} \times^{\prime \prime} V ; \mathbf{Z}\right) \quad \rightarrow \quad 0 \\
& \downarrow \rho \quad \downarrow \rho \quad \downarrow \rho \\
& H^{1}\left(\Omega_{U V} ; \theta\right) \quad \stackrel{e}{\rightarrow} \quad H^{1}\left(\Omega_{U V} ; \theta^{*}\right) \quad \rightarrow \quad H^{2}\left(\Omega_{U V} ; \mathbf{Z}\right)
\end{aligned}
$$

and taken $\phi=e^{-1} \rho c^{-1} k$ for any $k \in \operatorname{ker} \rho: H^{2}\left(U^{\prime \prime} \times^{\prime \prime} V ; \mathbf{Z}\right) \rightarrow H^{2}\left(\Omega_{U V} ; \mathbf{Z}\right)$.

LEMMA 3.1 Let $k$ generate the kernel of

$$
\rho: H^{2}\left(U^{\prime \prime} \times{ }^{\prime \prime} V ; \mathbf{Z}\right) \rightarrow H^{2}\left(\Omega_{U V} ; \mathbf{Z}\right),
$$

and set $\phi=e^{-1} \rho c^{-1} k$. The spin $n / 2$ Green's functions are then given by, for $n \geqslant-1$,

$$
\phi_{n}=(W \cdot Z)_{n+1} \cdot \phi \in H^{2}\left(U^{\prime \prime} \times^{\prime \prime} V ; \theta(-n-2,-n-2)\right) \text {. }
$$

Proof. This is simply a matter of evaluating the associated spacetime field. If we fix twistors $A^{\alpha}$ and $B^{\alpha}$ (and denote $W_{\alpha} A^{\alpha}$ by $W \cdot A$, etc.), the transition functions for the hyperplane section bundle on $\mathbf{P}^{*}$ (which has Chern class -1 ) are given by $(W \cdot A) /(W \cdot B)$ on $\{W \cdot A \neq 0\} \cap\{W \cdot B \neq 0\}$. We can therefore take

$$
\phi=\frac{1}{2 \pi i} \log \left(\frac{W \cdot A}{W \cdot B} \frac{\bar{B} \cdot Z}{\bar{A} \cdot Z}\right)
$$

and

$$
\phi_{n}=-\frac{1}{2(2 \pi i)^{2}} \frac{(-1)^{n}(n+1) !}{(W \cdot Z)^{n+2}} \log \left(\frac{W \cdot A}{W \cdot B} \frac{\bar{B} \cdot Z}{\bar{A} \cdot Z}\right) .
$$

To evaluate the field associated to this cohomology element, we use the usual twistor contour integral formulae $[14,11]$. The details are in [9]; the result for $n=0$, for example, is

$$
G(x, y)=\frac{1}{4 \pi^{2}} \frac{1}{(x-y)^{2}},
$$

which is indeed the usual Green's function for the free space wave operator.

The Green's function in (3.2) is singular for $x$ and $y$ null separated, and it is possible to see this directly in the twistor construction. If $U$ and $V$ are null disjoint, $\Omega_{U V}$ is a fiber bundle with base $U^{\prime \prime}$ and fiber $\left\{W_{\alpha} \in{ }^{\prime \prime} V\right.$ such that $\left.W_{\alpha} Z^{\alpha}=0\right\}$ for a fixed $Z^{\alpha} \in U^{\prime \prime}$. For $U$ and $V$ null disjoint, the fiber is contractible and it follows that $H^{2}\left(\Omega_{U V} ; \mathbf{Z}\right) \simeq H^{2}\left(U^{\prime \prime} ; \mathbf{Z}\right) \simeq \mathbf{Z}$. In the more general case, $H^{2}\left(\Omega_{U V} ; \mathbf{Z}\right) \simeq \mathbf{Z} \oplus \mathbf{Z}$. Since $H^{2}\left(U^{\prime \prime} \times^{\prime \prime} V ; \mathbf{Z}\right) \simeq \mathbf{Z} \oplus \mathbf{Z}$ for any $U$ and $V$,

$$
\operatorname{ker} \rho: H^{2}\left(U^{\prime \prime} \times^{\prime \prime} V ; \mathbf{Z}\right) \rightarrow H^{2}\left(\Omega_{U V} ; \mathbf{Z}\right)
$$

is nonvanishing if and only if the Green's function is well-behaved.

Lemma 3.2 Suppose $H^{\mathrm{l}}\left(\tilde{\Omega}_{U V} ; \mathbf{Z}\right) \simeq \mathbf{Z}$, and let $\tilde{k}$ generate this group. Then $\boldsymbol{\phi}$ in Lemma 3.1 satisfies $\pi^{*} \phi=i \tilde{k}$, where $i: H^{1}\left(\tilde{\Omega}_{U V} ; \mathbf{Z}\right) \rightarrow H^{1}\left(\tilde{\Omega}_{U V} ; \theta\right)$ is the usual injection and $\pi^{*} \phi$ is the pullback of $\phi$ to $\tilde{\Omega}_{U V}$. 
Proof. Consider the commutative diagram

$$
\begin{aligned}
& H^{1}\left(\tilde{\Omega}_{U V} ; \mathbf{Z}\right) \stackrel{i}{\rightarrow} H^{1}\left(\tilde{\Omega}_{U V} ; \theta\right) \stackrel{e}{\rightarrow} H^{1}\left(\tilde{\Omega}_{U V} ; \theta^{*}\right) \\
& \uparrow \pi^{*} \quad \uparrow \pi^{*} \\
& H^{1}\left(\Omega_{U V} ; \theta\right) \rightarrow H^{1}\left(\Omega_{U V} ; \theta^{*}\right)
\end{aligned}
$$

where the top row is exact. Since the hyperplane section bundle is trivial when pulled back to $\mathbf{T}$, it follows that $e \pi^{*} \phi=e\left(\tilde{\xi} \tilde{\xi}^{*-1}\right)=0$ on $\mathbf{T}$, so that there is a $\tilde{j} \in H^{1}\left(\tilde{\Omega}_{U v} ; \mathbf{Z}\right)$ with $i(\tilde{j})=\pi^{*} \phi$.

Since $c\left(\xi \xi^{*-1}\right)$ generates $\operatorname{ker} \rho: H^{2}\left(U^{\prime \prime} \times^{\prime \prime} V ; \mathbf{Z}\right) \rightarrow H^{2}\left(\Omega_{U V} ; \mathbf{Z}\right)$, it follows that if $\tilde{k}$ generates $H^{1}\left(\tilde{\Omega}_{U V} ; \mathbf{Z}\right)$, then $\pi^{*} \phi=i \tilde{k}$.

As in the paragraph following the proof of Lemma 3.2, it is not hard to see that $H^{1}\left(\tilde{\Omega}_{U V} ; \mathbf{Z}\right) \simeq \mathbf{Z}$ if $U$ and $V$ are null disjoint, and $H^{1}\left(\tilde{\Omega}_{U V} ; \mathbf{Z}\right)=0$ otherwise.

THEOREM 3.3. Let $U, V \subset \mathbf{M}$ be null disjoint. Then $H^{1}\left(\tilde{\Omega}_{U V} ; \mathbf{Z}\right) \simeq \mathbf{Z}$; let $\tilde{k}$ generate this group. The spin $n / 2$ Green's function on $U \times V$ then corresponds to the cohomology element

$$
(W \cdot Z)_{n+1} \cdot \tilde{k} \in H^{2}\left(U^{\prime \prime} \times^{\prime \prime} V ; \theta(-n-2,-n-2)\right)
$$

for $n \geqslant-1$.

Proof. Using Lemma 3.2, this is the same twistor function as $(W \cdot Z)_{n+1} \cdot \phi$. Now use Lemma 3.1.

4. Coupled Green's functions. In the coupled case, we will use Ward's deformed twistor space and then prove an analog to Theorem 3.3.

Throughout this section, let $E$ be a line bundle over a region $U^{\prime \prime} \subset \mathbf{P}$, and $F$ a line bundle over " $V \subset \mathbf{P}^{*}$, where $U$ and $V$ are null disjoint. Suppose further that the Chern classes of $E$ and $F$ are zero.

We now define complex manifolds $\tilde{T}=E(-1)$ and $\tilde{T}^{*}=F(-1)$, together with projections

$$
\pi: T^{\top} \rightarrow U^{\prime \prime} \text { and } \pi: T^{*} \rightarrow " V \text {, }
$$

and take $\tilde{\omega}_{U V}$ to be the inverse image of $\Omega_{U V}$ under these projections.

LEMMA. 4.1. $H^{1}\left(\tilde{\omega}_{U V} ; \mathbf{Z}\right) \simeq \mathbf{Z}$.

Proof. Since $\mathcal{T}$ is a deformation of $\mathbf{T}$ and $\mathcal{T}^{*}$ is a deformation $\mathbf{T}^{*}, \tilde{\omega}_{U V}$ is a deformation of $\tilde{\Omega}_{U V} \cdot H^{1}\left(\tilde{\omega}_{U V} ; \mathbf{Z}\right) \simeq H^{1}\left(\tilde{\Omega}_{U V} ; \mathbf{Z}\right)$ because the Chern classes of $E$ and $F$ vanish, and we have already noted that $H^{2}\left(\tilde{\Omega}_{U V} ; \mathbf{Z}\right) \simeq \mathbf{Z}$.

THEOREM 4.2. Let $\tilde{\kappa}$ generate $H^{1}\left(\tilde{\omega}_{U V} ; \mathbf{Z}\right)$. Then for $n \geqslant-1,(W \cdot Z)_{n+1} \cdot \tilde{\kappa}$ can be thought of as an element of $H^{2}\left(U^{\prime \prime} \times{ }^{\prime \prime} V ; E \times F(-n-2,-n-2)\right)$. This is the spin $n / 2$ Green's function, minimally coupled to $E$ in the first variable and to $F$ in the second.

Proof. Using the usual injection, we have $\tilde{\kappa} \in H^{1}\left(\tilde{\omega}_{U v} ; \theta\right)$. We have already remarked that this last group can be identified with $H^{1}\left(\tilde{\Omega}_{U V} ; \tilde{E} \times \tilde{F}\right)$, so

$$
(W \cdot Z)_{n+1} \cdot \tilde{\kappa} \in H^{2}\left(U^{\prime \prime} \times{ }^{\prime \prime} V ; \tilde{E} \times \tilde{F}(-n-2,-n-2)\right) .
$$


For $n \geqslant-1, \tilde{E}(-\mathrm{n}-2)$ has no global sections (since it has no global sections when restricted to any line), and Theorem 2.2 gives us

$$
H^{2}\left(\tilde{U}^{\prime \prime} \times{ }^{\prime \prime} \tilde{V} ; \tilde{E} \times \tilde{F}(-n-2,-n-2)\right) \simeq H^{2}\left(U^{\prime \prime} \times{ }^{\prime \prime} V ; E \times F(-n-2,-n-2)\right) \text {. }
$$

This completes the proof of the first part of the theorem. To see the second part. it suffices to note that $(W \cdot Z)_{n+1} \cdot \tilde{\kappa}$ varies holomorphically with the line bundles $E$ and $F$, and corresponds to the correct Green's function for $E$ and $F$ trivial, by Theorem 3.3

5. Concluding remarks. For Green's functions of negative helicity, there is difficulty finding suitable analogs to the expressions (3.1) which continue to satisfy

$$
\frac{\partial(W \cdot Z)_{n}}{\partial W_{\alpha}}=Z^{\alpha}(W \cdot Z)_{n+1},
$$

etc., for $n<-1$. It is possible to solve this problem using logarithms; the construction is rather messy and can be found in [9].

It is not clear whether or not the ideas we have presented can be extended to couple Green's functions to vector bundles of higher rank. The difficulty arises because there is no manifold $\widetilde{T}_{E}$ such that holomorphic functions from ${ }^{\top}{ }_{E}$ to $\mathbf{C}^{k}$ (where $k$ is the rank of the bundle) can be identified with sections of $E$ (as in the first paragraph of the proof of Theorem 4.2).

\section{REFERENCES}

1. M. F. Ativah, Green's functions for self-dual manifolds, J. Math. Anal. Appl. 7A (1981), 129-158.

2. M. F. Atiyah, N. J. Hitchin, V. G. Drinfeld and Yu. I. Manin, Construction of instantons, Phys. Lett. A 65 (1978). 185-187.

3. M. (j. Eastwood, On non-projective twistor cohomologv. Twistor Newsletter 10 (1980), 17.

4. M. G. Eastwood and M. L. Ginsberg, Duality in twistor theon, Duke Math. J. 48 (1981), 177- 196.

5. M. G. Eastwood, R. Penrose and R. O. Wells, Jr., Cohomology and massless fields, Comm. Math. Phys. 78 (1981). 305-351.

6. S. Gasioriwicz, Elementan particle phisics, Wiley, New York. 1966.

7. M. L. Ginsberg, A cohomological approach to scattering theorv, D. Phil. Thesis, Oxford, 1980.

8. Coupled propagators, Twistor Newsletter 13 (1981), 14-15.

9. Scattering theon and the geometn of multi-twistor spaces, Trans. Amer. Math. Soc. (to appear).

10. R. Godement. Topologie algébrique et théorie des faisceaux, Hermann, Paris, 1964.

11. L. P. Hughston and R. S. Ward (eds.). Advances in twistor theory, Research Notes in Math., no. 37 , Pitman, London, 1979.

12. R. Penrose, Twistor algehra, J. Mathematical Phys. 8 (1967), 345-366.

13. The structure of space-time. In: Batelle rencontres, 1967, pp. 121-235; Benjamin. New York, 1968.

14. Solutions of the zero-rest-mass equations, J. Mathematical Phýs. 10 (1969), 38-39.

15. Twistor theon', its aims and achievements, In: Quantum gravity: an Oxford symposium (eds. C. J. Isham, R. Penrose, D. W. Sciama), Clarendon Press, Oxford, 1975, pp. 268-407.

16. R. S. Ward, Curved twistor spaces, D. Phil. Thesis, Oxford, 1977.

17. On self-dual gauge fields, Phys. Lett. A. 61 (1977), 81-82.

Brasenose College, Oxford OXI 4AJ, England 